ARTICLE

DOI: $10.1038 / s 41467-017-00043-3$

OPEN

\title{
Dendritic mitoflash as a putative signal for stabilizing long-term synaptic plasticity
}

\author{
Zhong-Xiao Fu' ${ }^{1,2,3,4}$, Xiao Tan 2,3,4,5, Huaqiang Fang ${ }^{3,4}$, Pak-Ming Lau ${ }^{2,5}$, \\ Xianhua Wang ${ }^{3,4}$, Heping Cheng ${ }^{3,4}$ \& Guo-Qiang Bi ${ }^{1,2,6,7}$
}

Mitochondrial flashes (mitoflashes) are recently discovered excitable mitochondrial events in many cell types. Here we investigate their occurrence in the context of structural long-term potentiation (sLTP) at hippocampal synapses. At dendritic spines stimulated by electric pulses, glycine, or targeted glutamate uncaging, induction of SLTP is associated with a phasic occurrence of local, quantized mitochondrial activity in the form of one or a few mitoflashes, over a 30-min window. Low-dose nigericin or photoactivation that elicits mitoflashes stabilizes otherwise short-term spine enlargement into sLTP. Meanwhile, scavengers of reactive oxygen species suppress mitoflashes while blocking sLTP. With targeted photoactivation of mitoflashes, we further show that the stabilization of SLTP is effective within the critical 30-min time-window and a spatial extent of $\sim 2 \mu \mathrm{m}$, similar to that of local diffusive reactive oxygen species. These findings indicate a potential signaling role of dendritic mitochondria in synaptic plasticity, and provide new insights into the cellular function of mitoflashes.

\footnotetext{
${ }^{1}$ Hefei National Laboratory for Physical Sciences at the Microscale, University of Science and Technology of China, Hefei, 230027, China. ${ }^{2}$ School of Life Sciences, University of Science and Technology of China, Hefei, 230027, China. ${ }^{3}$ State Key Laboratory of Membrane Biology, Institute of Molecular Medicine, Peking-Tsinghua Center for Life Sciences, Peking University, Beijing, 100871, China. ${ }^{4}$ Beijing Key Laboratory of Cardiometabolic Molecular Medicine, Institute of Molecular Medicine, Peking-Tsinghua Center for Life Sciences, Peking University, Beijing, 100871, China. ${ }^{5}$ CAS Key Laboratory of Brain Function and Disease, University of Science and Technology of China, Hefei, 230027, China. ${ }^{6}$ CAS Center for Excellence in Brain Science and Intelligence Technology, University of Science and Technology of China, Hefei, 230027, China. ${ }^{7}$ Innovation Center for Cell Signaling Network, University of Science and Technology of China, Hefei, 230027, China. Zhong-Xiao Fu and Xiao Tan contributed equally to this work. Correspondence and requests for materials should be addressed to X.W. (email: xianhua@pku.edu.cn) or to H.C. (email: chengp@pku.edu.cn) or to G.-Q.B. (email: gqbi@ustc.edu.cn)
} 
S ynaptic plasticity is regarded as the neuronal basis of learning and memory ${ }^{1-3}$. It involves a series of subcellular signals and structural changes, including $\mathrm{Ca}^{2+}$ influx, activation of protein kinases, reorganization of the cytoskeleton, as well as synthesis and translocation of proteins ${ }^{4-7}$. Two distinct temporal phases of plasticity have been characterized: an early phase that involves signaling and structural changes within seconds to minutes after induction, and a late phase that involves enduring changes lasting for tens of minutes to hours ${ }^{8-10}$, yet the key process linking the two phases are less well defined. Like many other cellular events, these synaptic processes are energy-consuming, and thus likely to require the involvement of mitochondria that play a pivotal role in the cellular and synaptic energy supply ${ }^{11}$. More than just a powerhouse, the mitochondrion is also emerging as an important signaling organelle that plays an active role in regulating synaptic $\mathrm{Ca}^{2+}$ signaling ${ }^{12,13}$. In addition, mitochondrial dysfunction is known to result in synaptic plasticity defects and to be pivotal in various neurological disorders ${ }^{12,14,15}$

Recently, it has been shown that respiring mitochondria exhibit a dynamic activity known as "mitochondrial flash" or "mitoflash", a transient event comprising mitochondrial depolarization, reactive oxygen species (ROS) production, and alkalization in the matrix $^{16-21}$. Mitoflash activity is closely associated with cellular and whole-animal metabolic state ${ }^{22}$, and modulates neural progenitor cell differentiation and proliferation ${ }^{23}$ as well as somatic cell reprogramming ${ }^{24}$. Acting as a digital reporter of the aging process, the frequency of mitoflash in a young adults can also predict the lifespan of Caenorhabditis elegans ${ }^{21}$. Given that mitochondria constitute one of the most abundant organelles in neuronal processes, it is conceivable that such mitoflash events, if occurring at synapses, may also participate in activity-induced synaptic plasticity.

In the current study, we explore whether and how mitochondrial flashes (mitoflashes) participate in synaptic plasticity in cultured hippocampal neurons, a model system that has been used to identify important cellular mechanisms and computational rules of synaptic plasticity ${ }^{25-28}$. Specifically, we aim at determining whether activities that lead to functional and structural changes at the synapse can also cause mitoflash production in dendritic mitochondria, and, if so, whether such mitoflashes can reciprocally impact on the outcome of synaptic plasticity. Because of the heterogeneity of synaptic plasticity across individual synapses, we employ various stimulation paradigms used in previous studies of long-term potentiation (LTP) to induce lasting morphological enlargement of individual dendritic spines, a phenomenon termed structural long-term potentiation $(\text { sLTP })^{29-32}$. We find that induction of late-phase sLTP at synaptic spines is associated with a preceding increase in mitoflash frequency occurring in nearby dendritic shafts, whereas mitoflash activity remains unaltered during induction of only short-term spine enlargement. Artificially-eliciting mitoflashes stabilizes short-term spine enlargement and switches it into enduring sLTP. Furthermore, mitoflashes produce local ROS elevations on the micrometre scale, and ROS scavengers suppress mitoflash activity and impair late-phase sLTP expression, permitting only short-term spine enlargement. These results indicate that dendritic mitoflashes may be involved in short-term to long-term conversion of postsynaptic changes, and that may reflect a spatiotemporally specific, two-way communication between synaptic spines and dendritic mitochondria in hippocampal neurons.

\section{Results}

Dendritic mitoflashes in hippocampal neurons. To explore the potential involvement of mitoflashes in synaptic plasticity, we used cultured hippocampal neurons ${ }^{33}$ transfected with mitochondrial matrix-targeting circularly-permuted yellow fluorescent protein (mt-cpYFP) as a mitoflash biosensor ${ }^{16}$. In the spiny dendrites of these neurons, mitochondria of various lengths occupied dendritic shafts in single file (Fig. 1a and Supplementary Fig. 1), and in the vast majority of cases, each spine had a mitochondrion at its dendritic base ( $>90 \%)$ or was within $2 \mu \mathrm{m}$ of the nearest mitochondrion (99\%) (Supplementary Fig. 1g, h). These dendritic mitochondria remained largely stationary during the period of observation, in contrast to frequent movements of axonal mitochondria, which were much smaller in size (Supplementary Fig. 2). Importantly, dendritic mitochondria underwent spontaneous mitoflash activity: individual events occurred as sudden increases of mt-cpYFP fluorescence intensity, lasted for tens of seconds, and were confined to single organelles (Fig. 1a, b; Supplementary Movie 1). Parallel multiparametric measurements revealed that mt-cpYFP-reported mitoflash activity was accompanied by alkalinization reported by mt-pHTomato (Supplementary Fig. $3 \mathrm{a}-\mathrm{c}$ ). The rising phase of the mitoflash was coupled with a step-increase of mitoSOX signal (Supplementary Fig. 3d), with mitoSOX irreversibly reacts with various ROS and displays a relative selectivity for superoxide. Further, concurrent mitochondrial depolarization was visualized as a decreasing tetramethylrhodamine ethyl ester (TMRE) signal (Supplementary Fig. 3e). Thus, dendritic mitoflashes of hippocampal neurons each consists of a ROS burst, a transient $\mathrm{pH}$ rise, and a reversible depolarization, and thus reflects an electrical and chemical excitation at the single-organelle level, as is the case in other cell types $16,18,34$.

Dendritic mitoflashes accompanying sLTP at adjacent spines. To induce synaptic plasticity, we first used a chemical LTP (cLTP) induction paradigm ${ }^{25}$, which involved 5-min exposure to $100 \mu \mathrm{M}$ glycine and resulted in a long-term increase in the amplitude of miniature excitatory postsynaptic currents (Supplementary Fig. 4). We found that a prominent phasic increase in mitoflash frequency peaked at 20 min after glycine exposure (Fig. 1a-c). This increase was completely blocked by the N-methyl-Daspartate receptor (NMDAR) antagonist D-AP5 (Fig. 1c), indicating that the glycine-induced mitoflash activity requires early $\mathrm{Ca}^{2+}$ influx through NMDARs, as is the case with the induction of $\mathrm{CLTP}^{25}$ as well as other forms of $\mathrm{LTP}^{4,27,35}$.

Using co-transfection with actin-mCherry and mt-cpYFP, we were able to monitor the structural changes of dendritic spines together with mitoflash events through the entire process of cLTP induction. Consistent with previous studies of CLTP and electrically-induced $\mathrm{LTP}^{25,36}$, significant long-term morphological growth (termed structural LTP or sLTP hereafter) was observed in a subpopulation of dendritic spines $(\sim 30 \%)$ after glycine exposure, as evidenced by an increase of integrated actinmCherry fluorescence that peaked early and persisted for at least $50 \mathrm{~min}$ after glycine stimulation (Fig. 1d). Interestingly, this subpopulation of spines, but not those that remained stable $(\sim 57 \%)$, exhibited higher dendritic mitoflash activity during the 10-40 min period following glycine exposure (Fig. 1e, f). Careful examination further revealed that all spines that underwent sLTP were each coupled with one or a few local mitoflashes during this period (Fig. 1f, g). It was also notable that $\sim 13 \%$ spines decreased significantly in size following glycine treatment, and mitochondria adjacent to these spines also showed an elevated mitoflash frequency (Fig. 1g). Such spines might have undergone some form of long-term depression, in part influenced by sLTP in neighboring spines through heterosynaptic signaling ${ }^{37}$. These results suggest that dendritic mitoflashes may occur in the context of structural plasticity at dendritic spines. 
In addition to chemical stimulation, we further employed the classical high frequency electrical field stimulation (HFS, $3 \times 100$ pulses at $100 \mathrm{~Hz}, 20 \mathrm{~s}$ apart) (Supplementary Fig. 5a) to induce sLTP. We found that after HFS, out of 179 spines examined, $62(34.6 \%)$ spines showed long-term enlargement, 25 (14\%) spines showed only short-term enlargement, and $92(51.4 \%)$ spines were stable (Fig. 1h and Supplementary Fig. 12a). Again,

a

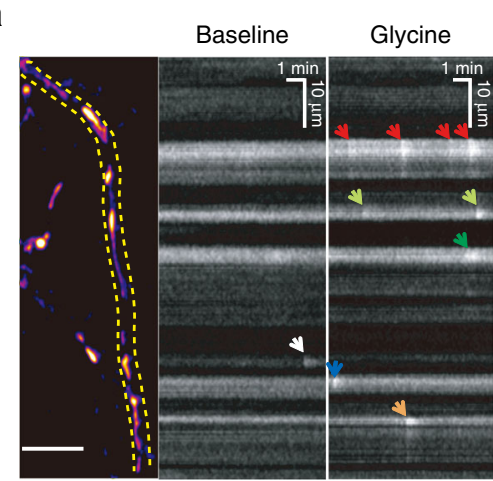

C

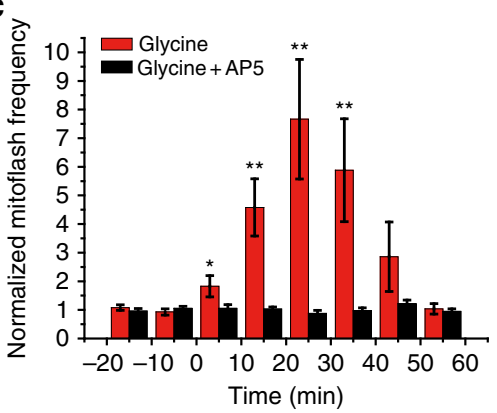

d

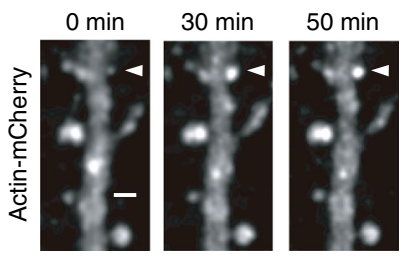

f

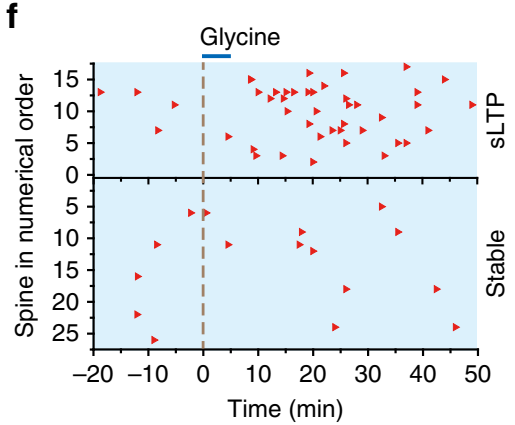

h

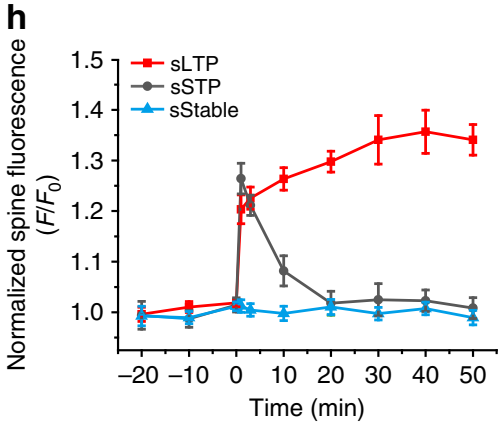

only spines underwent long-term enlargement were found to have increased local dendritic mitoflash frequency within a 30-min time window after HFS (Fig. 1i and Supplementary Fig. 5b). These results indicate that spine sLTP is generally associated with a phasic increase in local dendritic mitoflash frequency.

In order to precisely delineate the relationship between synaptic plasticity and mitoflashes, we then targeted individual

b

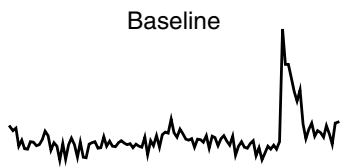

Glycine

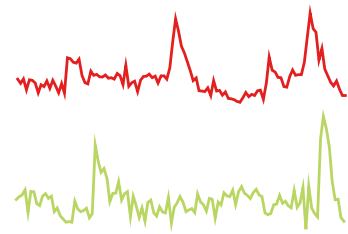

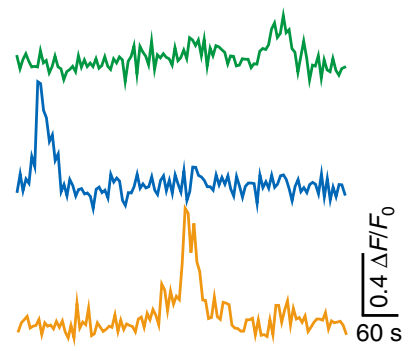

e

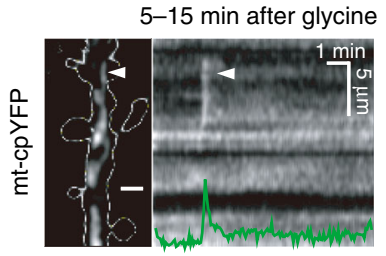

g

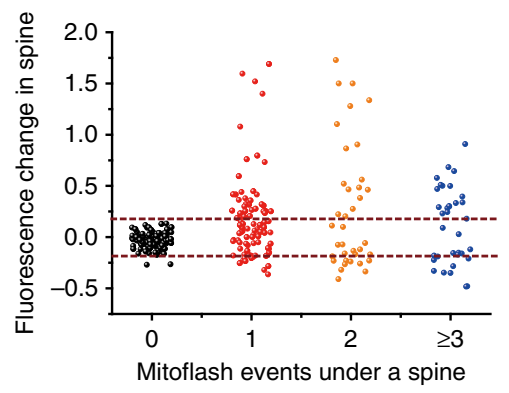

i

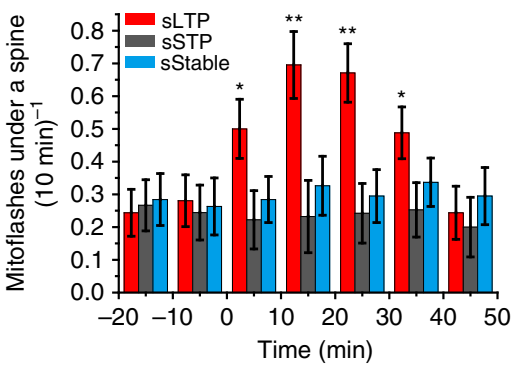


spines using two-photon photolysis of MNI-caged glutamate (4-methoxy-7-nitroindolinyl-caged-L-glutamate), similar to the method used to induce sLTP in hippocampal slices ${ }^{38,39}$. Three paradigms of stimulation were created by varying MNI-caged glutamate concentration and the number of uncaging laser pulses (Supplementary Fig. 6a). In the high concentration, strong uncaging (HS) paradigm, a train of 120 two-photon pulses $(720 \mathrm{~nm}, 2.5 \mathrm{~ms}, 8 \mathrm{~mW})$ at $2 \mathrm{~Hz}$ in the presence of $6-\mathrm{mM}$ MNIcaged glutamate induced sLTP at targeted spines, but not at neighboring spines (Fig. 2a, c), in agreement with previous reports ${ }^{8,40}$. Kinetic analysis revealed a biphasic change of spine size, characterized by an early peak followed by a gradual $\sim 16 \%$ decay, before stabilization at an elevated plateau. In a representative example, simultaneous mitoflash imaging detected seven events occurring in the mitochondrion immediately beneath the stimulated spine during the 10-40 min period after uncaging, but only one event in a nearby mitochondrion during the same period (Fig. 2b). Overall, during the 10-40 min after glutamate uncaging, a robust transient increase of mitoflash events occurred in the mitochondria underlying stimulated spines but not in those beneath nearby, unstimulated spines (Fig. 2e), the latter did not undergo sLTP (Fig. 2d and Supplementary Fig. 12b). It is noteworthy that no change in the amplitude or kinetics of mitoflashes was found after uncaging stimulation (Supplementary Fig. $6 \mathrm{~b}-\mathrm{d})$. This result indicates that spine activity-triggered mitoflashes and spontaneous ones are indistinguishable, consistent with the notion that mitoflash, if serving a signaling role, would mainly operates via frequency-modulation ${ }^{21,22}$.

With a lower concentration ( $2 \mathrm{mM})$ of MNI-caged glutamate, strong single spine-targeted glutamate uncaging (low-concentration strong stimulation, LS paradigm) (Supplementary Fig. 6a) elicited a slowly developing sLTP that exhibited a slow monophasic enlargement in $49 \%$ of spines (Fig. $2 \mathrm{~g}$ and Supplementary Fig. 12c). An increase in mitoflash frequency was again seen only in mitochondria next to the enlarged spines 10-40 min after uncaging, but not in those adjacent to stable spines (Fig. 2h). Moreover, in both the HS and LS paradigms, the number of mitoflash events beneath a stimulated spine appeared to positively correlate with the degree of spine size enlargement (Fig. 2f, i). These set of experiments illustrates a tight spatiotemporal specificity between sLTP at single spines and mitoflash activation in dendritic shafts.

The induction and expression of sLTP at the dendritic spine involve a complex signaling cascade including the early processes such as $\mathrm{Ca}^{2+}$ ion influx through NMDA receptors and activation of $\mathrm{Ca}^{2+} /$ calmodulin-dependent protein kinase II (CaMKII) and small GTPase $6,41,42$. The blocking effect of D-AP5 already showed an essential role of $\mathrm{Ca}^{2+}$ influx through NMDARs. However, since mitoflash activation peaked at $\sim 20-30 \mathrm{~min}$ after spine stimulation, it appeared unlikely that $\mathrm{Ca}^{2+}$ transients directly trigger the delayed mitoflash response. To evaluate the role of signaling events downstream of $\mathrm{Ca}^{2+}$, we performed the same uncaging experiment in the presence of KN93, an inhibitor of CaMKII that is known to be crucial for LTP in hippocampal cultures as well as in other systems ${ }^{28,35}$. Our results showed that both sLTP and sLTP-linked mitoflash activation were blocked by KN93, but not by the control compound KN92 (Fig. 3a, b). Similar results were obtained with two other CaMKII inhibitors, KN62 and autocamtide-2-related inhibitory peptide (AIP) (Fig. 3c, d). Thus, CaMKII activation as a key step in LTP induction is required for mitoflash activation.

Mitoflashes during short- to long-term transition of sLTP. The HS paradigm-induced sLTP might be considered as a two-step process, a fast, early-phase enlargement followed by a slowly developing stabilization into a sustained structural change. Plasticity-triggered mitoflashes were found in experimental paradigms when sLTP was fully expressed. To evaluate mitoflash response to the early phases of spine plasticity, we used a weak uncaging paradigm (40 pulses at $2 \mathrm{~Hz}, \mathrm{HW}$ ) (Supplementary Fig. 6a), which was shown to induce only short-term enlargement of targeted spines ${ }^{8}$. Indeed, while spines responded with a $\sim 1.8$ fold peak enlargement, comparable to those in the HS paradigm, they completely recovered in $\sim 40 \mathrm{~min}$ (Fig. $4 \mathrm{a}, \mathrm{b}$ and Supplementary Fig. 12d), indicating that such spine enlargement failed to stabilize. Surprisingly, mitochondria at the bases of targeted spines showed no significant increase in mitoflash frequency (Fig. 4c), in contrast to their responses in the HS and LS paradigms. Thus, although one cannot exclude the possibility that spine plasticity-triggered phasic mitoflashes are an epiphenomenon accompanying synaptic changes, it is worthwhile to evaluate the potential role of mitoflash as a putative signal to stabilize spine enlargement after initial induction and to support the shortto long-term transition of sLTP.

One way to directly test our hypothesis is to artificially activate mitoflashes within the critical time-window prior to spine restoration, i.e., $10-40 \mathrm{~min}$ after initial induction of spine enlargement. For this purpose, we first employed the mitoflash activator nigericin, a $\mathrm{K}^{+} / \mathrm{H}^{+}$antiporter, which has recently been shown in muscle cells to enhance mitoflash activity without disturbing the mitochondrial membrane potential when applied at low concentrations ${ }^{18}, 43$. We found that $50 \mathrm{nM}$ nigericin rapidly and persistently augmented mitoflash frequency among the whole population of dendritic mitochondria in hippocampal neurons (Fig. 4c). More importantly, combination of the HW paradigm of single spine activation with immediate nigericin application resulted in persistent sLTP alongside mitoflash

Fig. 1 Dendritic mitoflashes occurring in chemical and electrical LTP induction. a Dendritic mitoflash activity before and after glycine stimulation. Left: mtcpYFP fluorescence revealing mitochondria in a dendritic segment. Dashed lines delimit the boundaries of the segment. Scale bar, $10 \mu \mathrm{m}$. Middle and right: kymographs showing discrete mitoflashes (marked by arrows) prior to and 20-29 min after glycine treatment. b Time-courses of mitoflashes shown in a. c Phasic increase in mitoflash frequency after glycine stimulation ( $100 \mu \mathrm{M}$ applied at time zero for $5 \mathrm{~min})$. Note that this increase was abolished by D-AP5. $n=10-12$ neurons from seven batches for each group. Unless otherwise stated, error bars in all figures hereafter report s.e.m. ${ }^{\star} P<0.05$, ${ }^{\star \star} P<0.01$ (paired $t$-test, after glycine vs. baseline). d Dendritic segment visualized by actin-mCherry at 0, 30, and 50 min after glycine treatment. Images are maximal projections of corresponding Z-stacks. White arrowheads mark a spine undergoing SLTP. Scale bar, $2 \mu \mathrm{m}$. e Mitoflash activity in the same dendrite as in d. Left: morphology of dendritic shaft and spines outlined by dashed lines overlaid on an mt-cpYFP fluorescence image. Scale bar, $2 \mu \mathrm{m}$. Right: kymograph and time-course (green) of a mitoflash event at the base of the marked spine in $\mathbf{d}$, occurring at $\sim 8 \mathrm{~min}$ after glycine stimulation. $\mathbf{f}$ Raster plot of mitoflash occurrence (red triangles) beneath 44 spines located on seven dendritic branches of a neuron, bisected into sLTP and stable groups according to sizechanges after glycine stimulation (blue bar). $\mathbf{g}$ Relationship between spine size-change and the number of corresponding mitoflash events occurring $0-50$ min after glycine treatment. Size-changes were quantified as mean actin-mCherry fluorescence intensity at 30, 40 , and 50 min, normalized to that prior to stimulation. Dashed lines mark 2 SD of the fluctuation under basal conditions. $n=9$ neurons from seven batches. $\mathbf{h}$ Long-term spine morphological changes induced by high frequency field electrical stimulation. $n=179$ spines in eight neurons from four batches. $\mathbf{i}$ Mitoflash activity in dendritic mitochondria corresponding to the spines studied in $\mathbf{h} .{ }^{\star} P<0.05,{ }^{\star \star} P<0.01$ (paired $t$-test, after electrical stimulation vs. baseline) 
a
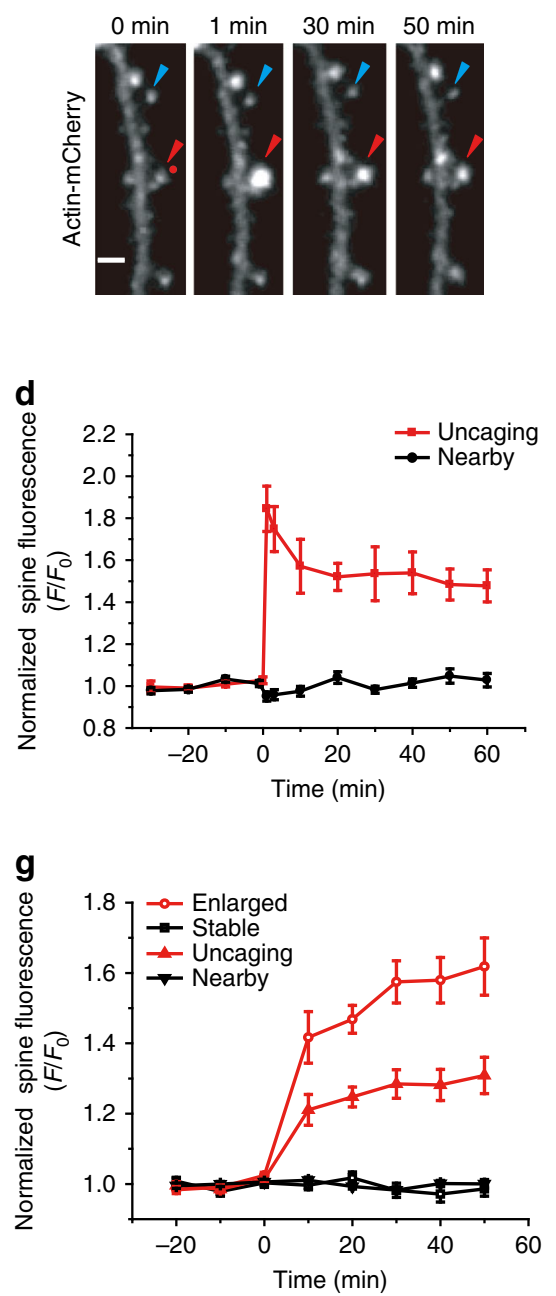

b
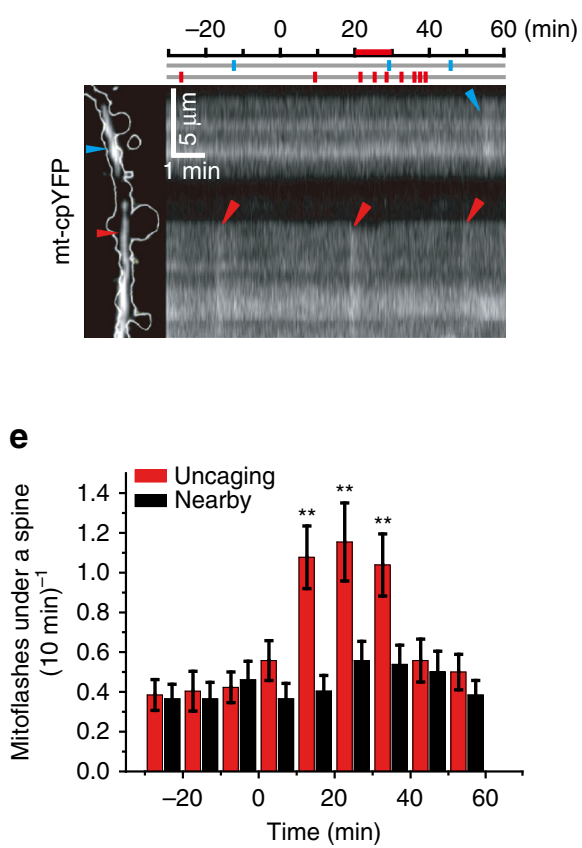

h

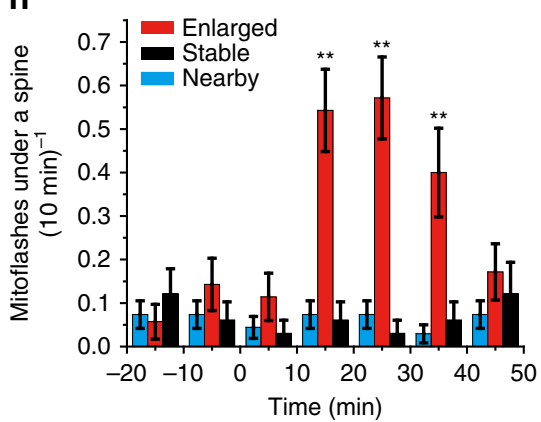

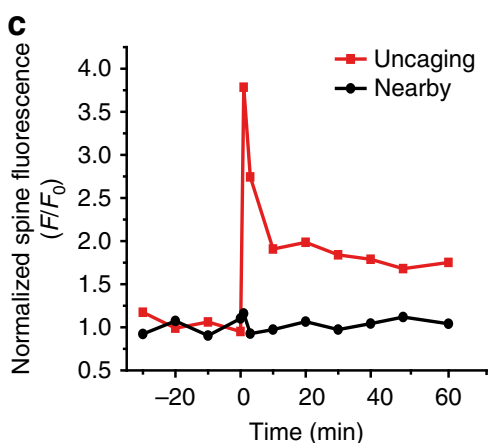
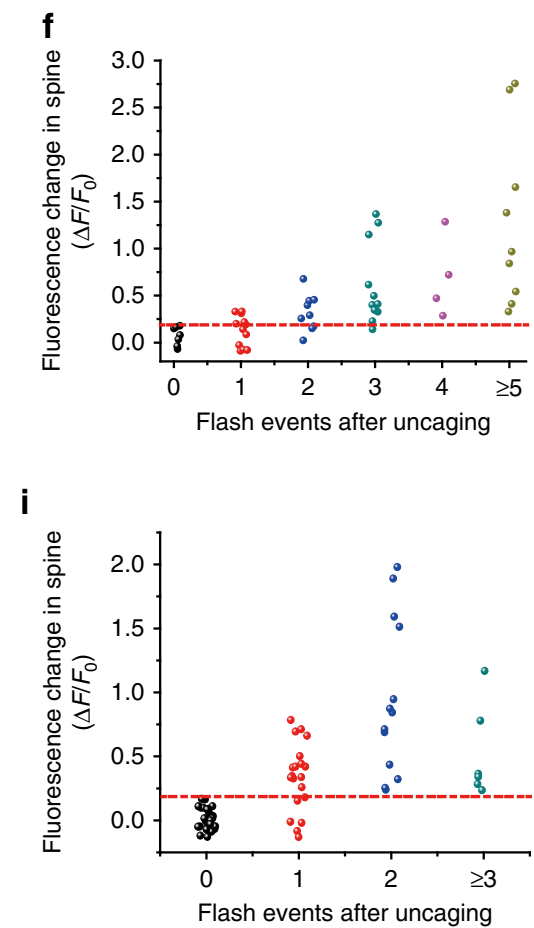

Fig. 2 Dendritic mitoflashes occurring in SLTP induced by single-spine-targeted glutamate uncaging. a Sequential view of spine enlargement following the "HS" two-photon uncaging paradigm ( $720 \mathrm{~nm}$ at $8 \mathrm{~mW}, 120$ pulses of $2.5 \mathrm{~ms}$ at $2 \mathrm{~Hz}$, with $6 \mathrm{mM} \mathrm{MNI-caged} \mathrm{L-glutamate.} \mathrm{See} \mathrm{also} \mathrm{Supplementary} \mathrm{Fig.} 6 a$ ). Red dot (at $0 \mathrm{~min}$ ) denotes the uncaging spot placed close to a spine. Red and blue arrowheads mark this target spine and a nearby spine, respectively. Scale bar, $2 \mu \mathrm{m}$. b Local mitoflash activity beneath the spines. Top inset: raster plot of mitoflashes facing the target spine (red ticks) and a nearby spine (blue tick) during a 90-min period. Left: White lines outline the dendritic shaft and spines overlaid on the mt-cpYFP image of dendritic mitochondria. Right: kymograph showing mitoflashes between 20 and 30 min after glutamate uncaging. c Changes in actin-mCherry fluorescence of the target and nearby spines.

d Statistics of SLTP induced in the HS paradigm. $n=51$ spines in ten neurons from four batches for each group. e Time-dependent changes of mitoflash frequency corresponding to $\mathbf{d}\left({ }^{\star \star} P<0.01\right.$; paired $t$-test). $\mathbf{f}$ Relationship between mitoflash number and spine size change. Data from the same experiments as in $\mathbf{d}$ and $\mathbf{e}$. $\mathbf{g}$-i Relationship between SLTP at individual spines and local mitoflash events, as in $\mathbf{d}-\mathbf{f}$, except that the LS paradigm was used (see also Supplementary Fig. 6a). $n=67$ for target and nearby spines in 18 neurons from six batches. The target spines were further divided into responding (open circles, size change $>2$ SD of basal fluctuation, $n=33$ spines in 15 neurons from six batches) and non-responding groups (open squares, $n=34$ spines in 18 neurons from six batches). Note that no change in mitoflash frequency was found in non-responding spines ( ${ }^{\star \star} P<0.01$; paired $t$-test)

activation, suggesting that the artificially-elicited mitoflashes might indeed contribute to the conversion of otherwise shortterm spine enlargement into sLTP (Fig. $4 \mathrm{a}, \mathrm{b}$ and Supplementary Fig. 12d).

Next, we modified a mitochondrial photo-excitation protocol $^{44}$ (see Methods, and Supplementary Figs. $3 \mathrm{f}-\mathrm{j}, 7$ ) to elicit a photoactivated mitoflash (PA-mitoflash) at a precisely designated time and location. In this experimental setting, two trains of femtosecond laser pulses $(80 \mathrm{MHz}, 720 \mathrm{~nm}, 10 \mathrm{~mW}, 1 \mathrm{~ms}$; delivered at a 30-s interval) were targeted to a designated mitochondrion. The success rate of PA-mitoflash activation was $\sim 50 \%$ and cases of failure were used as experimental controls (Fig. $4 \mathrm{e}, \mathrm{f})$. In contrast to Shi et al. ( $80 \mathrm{MHz}, 810 \mathrm{~nm}, 10-17 \mathrm{~mW}$, $100 \mathrm{~ms}-3 \mathrm{~s})^{44}$, our laser stimulation by itself did not induce any detectable changes in local ROS or calcium concentration (Supplementary Figs. 3j, 7, 9), nor did it cause any mitochondrial swelling or fragmentation regardless of mitoflash activation (Supplementary Fig. 7). These PA-mitoflashes, as detected by multiple reporters including TMRE and mitoSOX, were indistinguishable from spontaneous and nigericin-triggered mitoflashes by virtues of amplitude and duration (Supplementary Fig. 3 and Supplementary Table 1). Strikingly, when combined with one or more early PA-mitoflashes (at 10 and/or 20 min after uncaging stimulation) (Fig. $4 \mathrm{~d}$ ), the $\mathrm{HW}$ paradigm evoked robust sLTP (Fig. 4e, f and Supplementary Fig. 12e). In contrast, no such sLTP was induced if the same laser pulses failed to elicit a PA-mitoflash (Figs. 4e, f and Supplementary Fig. 12e), or if the PA-mitoflash events were elicited late (30 and/or $40 \mathrm{~min}$ after 
a

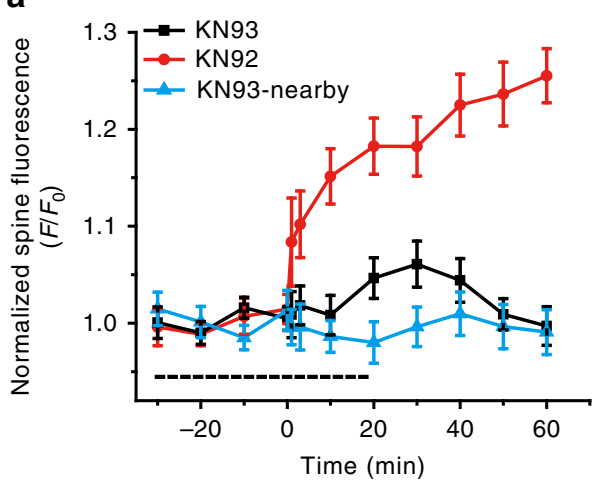

C

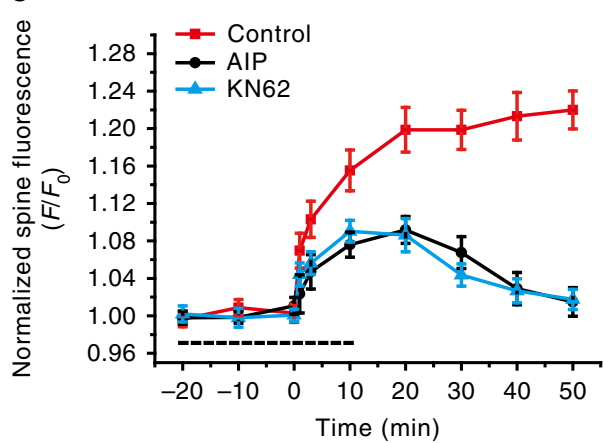

b

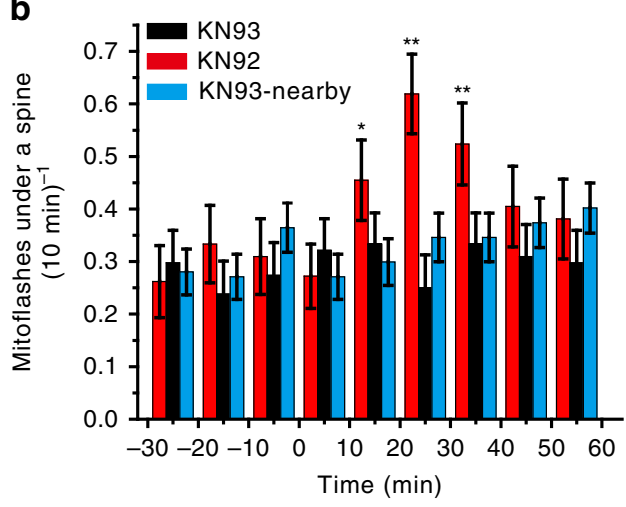

d

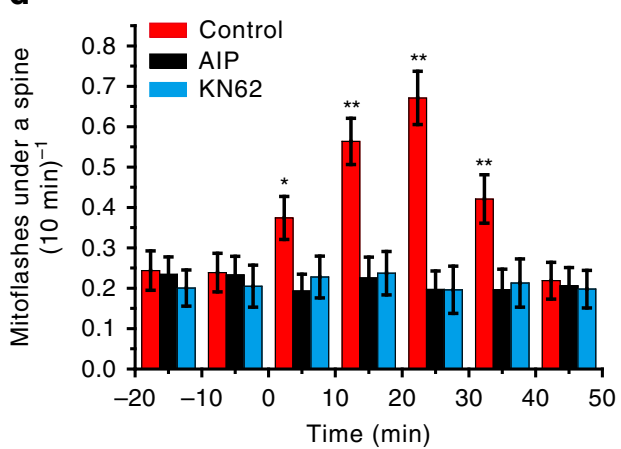

Fig. 3 Dendritic mitoflashes occurring in SLTP depends on CaMKII activation. a Effects of the CaMKII inhibitor KN93 (10 $\mu$ M) and its analog KN92 (10 $\mu$ M) on SLTP induced by glutamate uncaging in the LS paradigm (see Supplementary Fig. 6a). Dashed line indicates the presence of KN93 or KN92. $n=6$ neurons in four batches for the KN92 and KN93 groups. b Glutamate-uncaging-induced mitoflash frequency changes corresponding to the data in $\mathbf{a}$. ${ }^{\star} P<0.05 ;{ }^{\star \star} P<0.01$; paired $t$-test vs. baseline. c Effects of the CaMKII inhibitors AIP $(10 \mu \mathrm{M})$ and KN62 (10 $\mu$ M) on sLTP induced by glutamate uncaging in the LS paradigm. $n=6$ neurons in three batches for AIP groups, $n=5$ neurons in three batches for KN62 groups, $n=7$ neurons in three batches for control groups. Dashed line indicates the presence of AIP or KN62. d Glutamate uncaging-induced mitoflash activity was blocked by AIP and KN62.

Data correspond to those in $\mathbf{c} .{ }^{\star} P<0.05$; ${ }^{\star} P<0.01$; paired $t$-test vs. baseline

uncaging) when the recovery from the early-phase spine enlargement was essentially complete (Fig. $4 \mathrm{f}$ and Supplementary Fig. 12e). Thus, dendritic mitoflashes evoked within a critical time-window appear to be effective in stabilizing spine size and permitting the full expression of sLTP. These results are consistent with a signaling role for mitoflash in the short-term to long-term transition of sLTP.

Local ROS signaling in a mitoflash. We hypothesized that diffusive messengers might emanate from the flashing mitochondria whereby dendritic mitoflashes drive the full induction of sLTP. We therefore measured local, cytosolic ROS (reported by 2',7'-dichlorofluorescein (DCF)), calcium (by GCaMP6f), and ATP (by magnesium green and PercevalHR) concentrations during mitoflashes. Our results showed that mitoflashes were accompanied by stepwise increases of cytosolic DCF signals that peaked in about $10 \mathrm{~s}$ and was spatially-graded over a distance of about $2 \mu \mathrm{m}$ (Supplementary Fig. 8), indicating diffusion of mitoflash ROS into the cytosol (Fig. 5a, b). On the other hand, we detected no appreciable changes in local calcium and ATP concentrations during mitoflashes (Supplementary Figs. 9, 10). This does not argue against the importance of general ATP production in sLTP. In fact, inhibiting ATP synthesis with FCCP or other ETC inhibitors severely impaired spine actin dynamics and sLTP (Supplementary Fig. 11). However, it is unlikely that mitoflashes support sLTP as burst of ATP energy supply. Because recent studies have implicated ROS signaling in synaptic plasticity ${ }^{45}, 46$, we examined possible effects of the ROS scavengers Tiron and
mitoTEMPO in glutamate uncaging-induced sLTP (Fig. 5c-e). Strikingly, both scavengers suppressed the phasic increase in mitoflash frequency in the HS paradigm (Fig. 5e), and effectively abolished sLTP, leaving only short-term spine enlargement (Fig. 5d and Supplementary Fig. 12f). These results are consistent with an involvement of mitoflash ROS in sLTP.

Further, using local PA-mitoflashes, we examined the spatial pattern of mitoflash signaling in sLTP. For this purpose, we elicited PA-mitoflashes at various distances from selected spines at 10 and $20 \mathrm{~min}$ after $\mathrm{HW}$ uncaging stimulation applied to the spines (Fig. 6a-d). We found that the mitoflash signaling in terms of stabilizing spine enlargement was highly confined to within only a few micrometres. Within this short distance, the farther away the PA-mitoflashes were, the less the spine growth could be sustained. Quantitatively, the degree of persistent spine enlargement fitted an exponential decay function of the distance between spine and PA-mitoflash, yielding a length constant of $\sim 2 \mu \mathrm{m}$ (Fig. 6e), similar to the range of cytosolic diffusion of mitoflash ROS as indicated by DCF signals. Although these observations do not exclude the possibility that the ROS elevation is an epiphephenomon of mitochondrial activity, they are consistent with the scenario that local cytosolic ROS transient in a mitoflash provides a signal for stabilizing structural plasticity at the spine.

\section{Discussion}

We present two findings suggesting a previously unknown interplay between synaptic plasticity in the form of sLTP and dynamic activity of dendritic mitochondria in the form of 
a

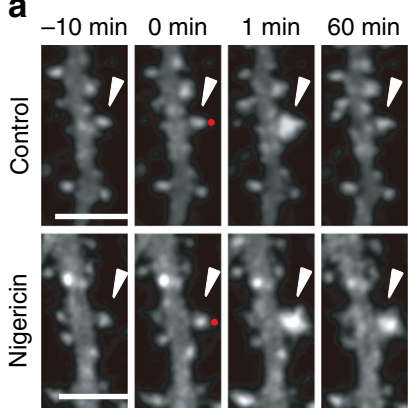

b

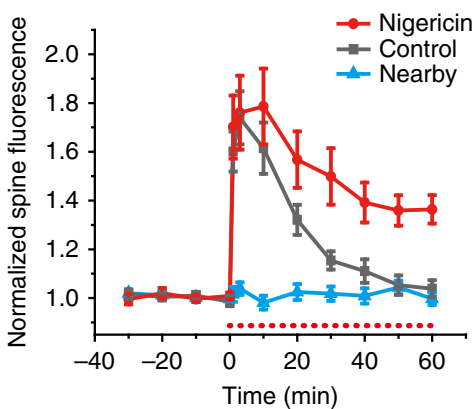

C

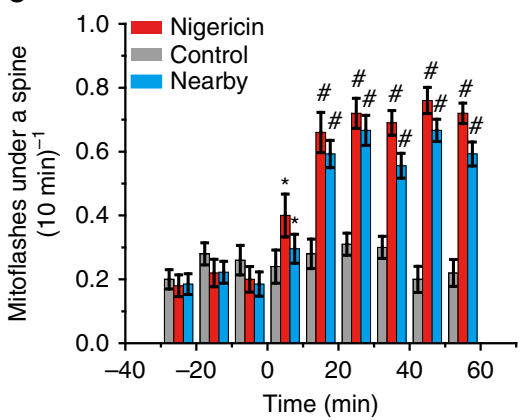

d

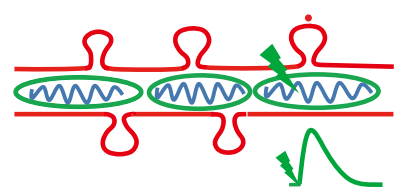

Control : HW-uncaging

$\Omega$ Spine Mitochondrion

- Glutamate uncaging site

2P-laser pulse

Photoactivated mitoflash

1 2P-uncaging of glutamate
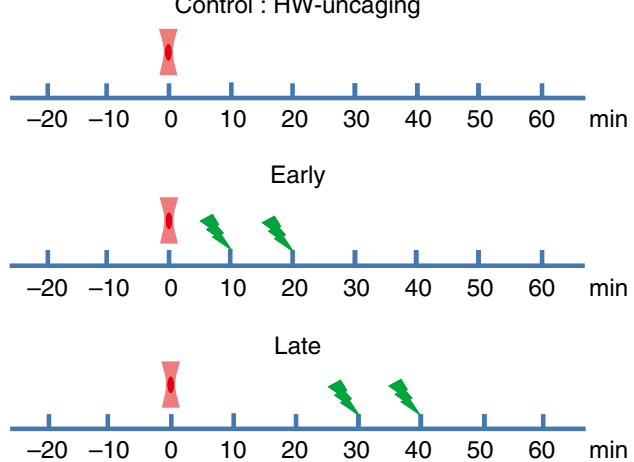

e

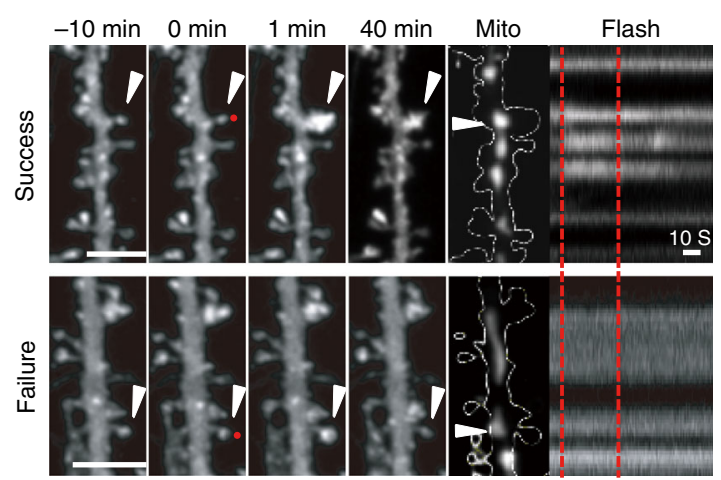

f

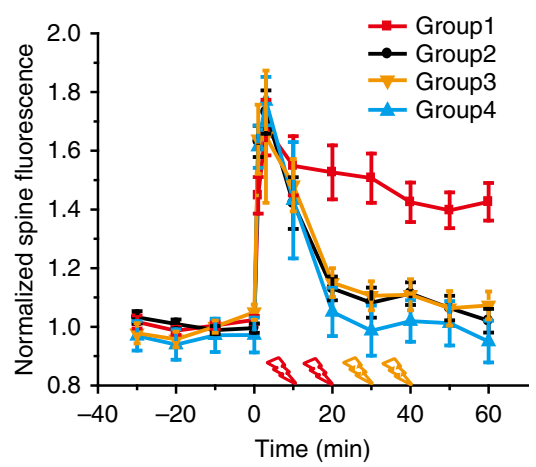

Fig. 4 Artificially-eliciting dendritic mitoflashes converts short-term spine enlargement into SLTP. $\mathbf{a}$, b Short-term spine enlargement induced by local glutamate uncaging using the HW paradigm (see also Supplementary Fig. 6a) and its conversion to sLTP by nigericin ( $50 \mathrm{nM}$ ). Representative examples and statistics are shown in $\mathbf{a}$ and $\mathbf{b}$, respectively. Arrowheads in $\mathbf{a}$ denote uncaging target spines and dotted line in $\mathbf{b}$ the period of nigericin application. Note that nigericin did not alter the magnitude of enlargement at target spines and the size of nearby spines. Red dots (at 0 min) denote the uncaging spots placed close to spines. Scale bars, $5 \mu \mathrm{m}$. For controls, $n=51$ spines on nine dendrites in six neurons. For nigericin and nearby groups, $n=45$ spines on eight dendrites in six neurons. c Dendritic mitoflash events corresponding to the spines in $\mathbf{b}$. Note that nigericin evoked greater, sustained mitoflash activity. ${ }^{\star} P<0.05,{ }^{*} P<0.01$; paired $t$-test compared to baseline. $\mathbf{d}$ Experimental protocol with photoactivated mitoflashes (PA-mitoflashes). e Representative examples showing that PA-mitoflashes stabilized otherwise short-term spine enlargement into SLTP (upper row), whereas laser pulses per se in failed attempts were ineffective (lower row). Horizontal arrowheads denote the target mitochondria for photoactivation. Vertical dashed lines mark the timing of laser pulses. Scale bars, $5 \mu \mathrm{m}$. f Statistics. Group 1: at least one PA-mitoflash evoked at 10 or 20 min; Group 2: no PA-mitoflash evoked; Group 3: at least one PA-mitoflash evoked at 30 or $40 \mathrm{~min}$; Group 4: no laser stimulation. $n=13$ neurons and ten batches for group 1 (61 spines) and group 2 (53 spines), and $n=5$ neurons and five batches for group 3 (28 spines) and group 4 (30 spines)

mitoflashes (Fig. 6f). Firstly, early signaling events during sLTP induction in a spine cause a phasic increase in mitoflash frequency in adjacent dendritic mitochondria, regardless whether the induction is through glycine treatment or high-frequency electrical stimulation or single-spine-targeted focal glutamate uncaging. This finding provides direct evidence that dendritic mitochondria can act as an organelle biosensor capable of decoding the complex cellular signal originated from the spine. It is clear that key signals for LTP induction, including $\mathrm{Ca}^{2+}$ influx through NMDARs and subsequent CaMKII activation ${ }^{2,4}, 6$, participate in this spine-to-mitochondrion signaling. Basal ROS signal is also involved because ROS scavengers effectively inhibit the sLTP-linked mitoflash activity. Albeit obligatory, the transient $\mathrm{Ca}^{2+}$ signal and CaMKII activation are unlikely the direct trigger, because the synaptic activity-linked mitoflashes occur within a broad 30 min window after synaptic stimulation. One possibility is that downstream processes responsible for spine enlargement, such as receptor recruitment and cytoskeletal reorganization, consume more energy and thereby alter metabolic state in nearby mitochondria. Indeed, it has been shown that, in other types of 
cells, mitoflash frequency varies depending on cellular metabolic state $^{22,47}$. Alternatively, the delayed mitoflash activation might be triggered by metabolically independent signals occurring late in the sLTP process.

Secondly, we found that dendritic mitoflashes appear to act reciprocally on the synapse, to stabilize spine enlargement and switch on the full expression of sLTP. Phasic increase of mitoflash activity is associated with full-fledged sLTP, but not when only a short-term enlargement is expressed, and this is true for all the chemical, electrical, and photolytic paradigms of synaptic stimulation. Manipulations that inhibit mitoflashes prevent sLTP without affecting the expression of short-term spine growth, suggesting that the mitoflash-sLTP link is causal and specific. Conversely, when combined with weak stimulations, artificially activating the mitoflash, with either chemical stimulation or photo-excitation, is sufficient to stabilize otherwise short-term spine enlargement into long-term sLTP. With precise photoactivation methods, we demonstrate that this mitochondrion-tospine signaling displays high spatiotemporal specificity: PA-mitoflashes are effective in supporting sLTP only if they are evoked during the early time window after spine stimulation $(<30 \mathrm{~min})$ and within a distance of a few micrometres. These results suggest that, in addition to being a biosensor, the mitochondrion has the potential to act as a signaling organelle and play a role in the signaling cascade of synaptic plasticity in hippocampal neurons.

Alternatively, mitochondrial activity may play a permissive role in sLTP as the mitochondrion in close proximity to a spine

\section{b}
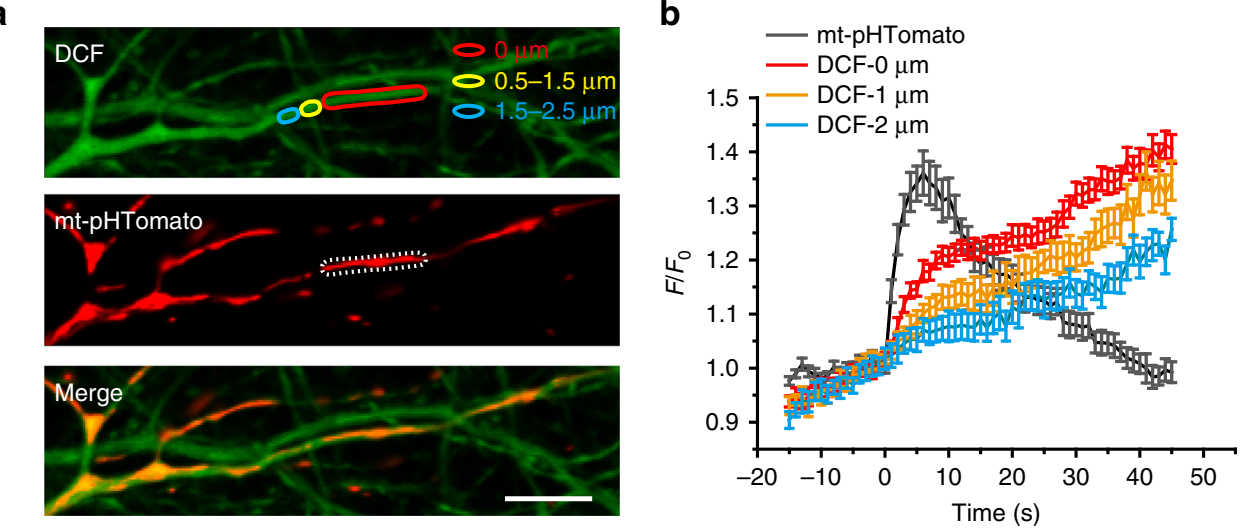

C
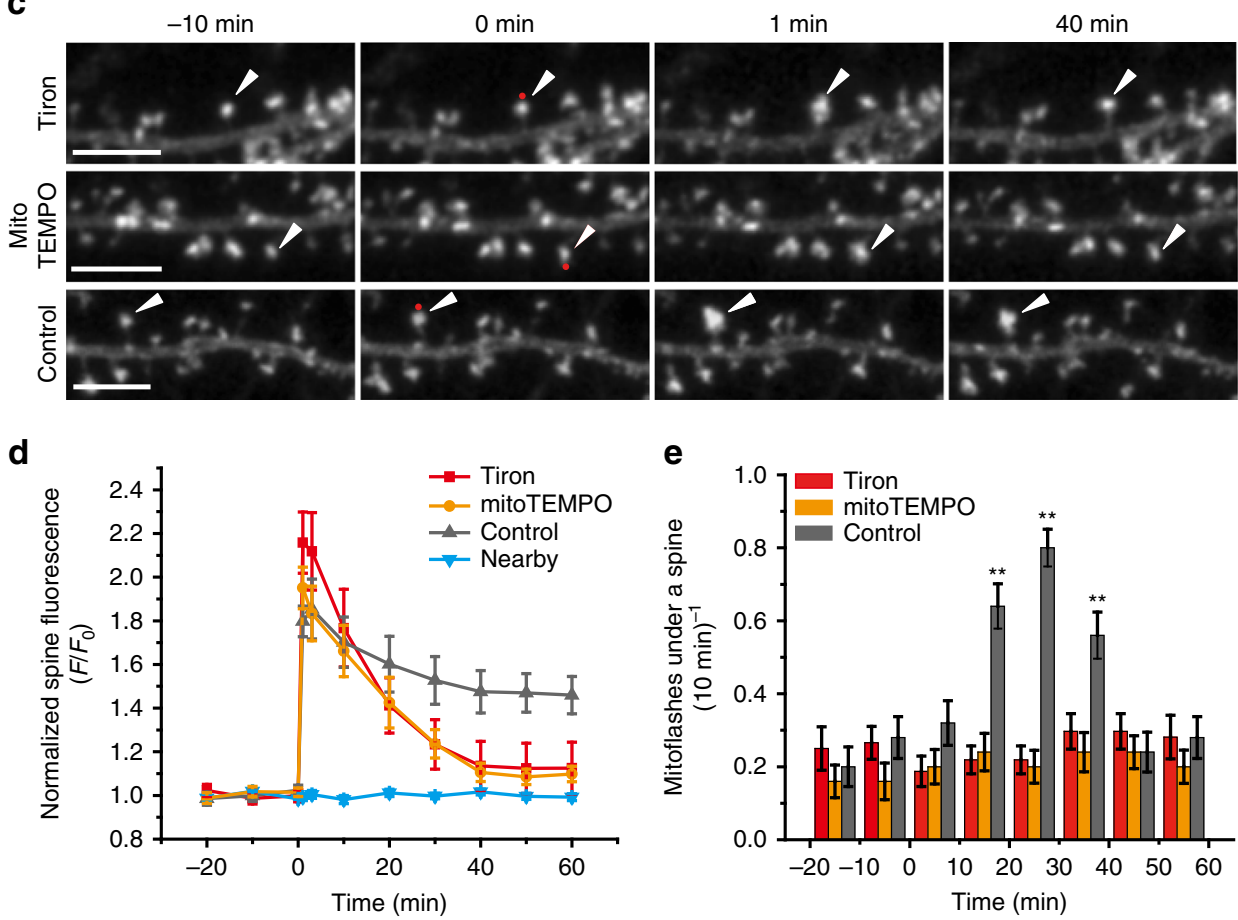

Fig. 5 Local cytosolic ROS signaling during mitoflashes. a, b Representative images showing cytosolic DCF signal for ROS detection (upper panel), mt-pHTomato signal for mtioflash detection (middle panel), and their merged image (bottom panel). Dashed line in the middle panel delimits a spontaneously flashing mitochondrion, and local DCF signals were measured in regions of interest marked in the upper panel, at different distances from the mitoflash. Scale bar, $5 \mu \mathrm{m}$. b Time courses of local cytosolic DCF signals during mitoflashes. $n=72$ events. See also Supplementary Fig. 8 . c Representative examples showing inhibitory effects of the ROS scavengers, Tiron and mitoTEMPO, on SLTP induced in the HS paradigm. Red dots (at 0 min) denote the uncaging spots placed close to spines. Arrowheads denote uncaging target spines. Scale bars, $5 \mu \mathrm{m}$. d Time courses of sLTP induction under different conditions. $n=7$ neurons and 4 batches for Tiron ( 45 spines), $n=7$ neurons and four batches for mitoTEMPO ( 32 spines), and $n=7$ neurons and four batches for control (48 spines) and nearby groups (48 spines). e Time courses of dendritic mitoflash frequency corresponding to $\mathbf{d}$. The nearby group is omitted for clarity. ${ }^{\star} P<0.01$ vs. baseline 
undergoing sLTP are providing the necessary energy required for this process. In such a scenario, the mitoflashes could simply reflect bursts of energy production in response to cellular stress due to increased energy demand from the changing spine. However, measurements with both magnesium green and percevalHR failed to reveal any fluctuations in local ATP concentration during mitoflashes. In contrast, mitoflashes are
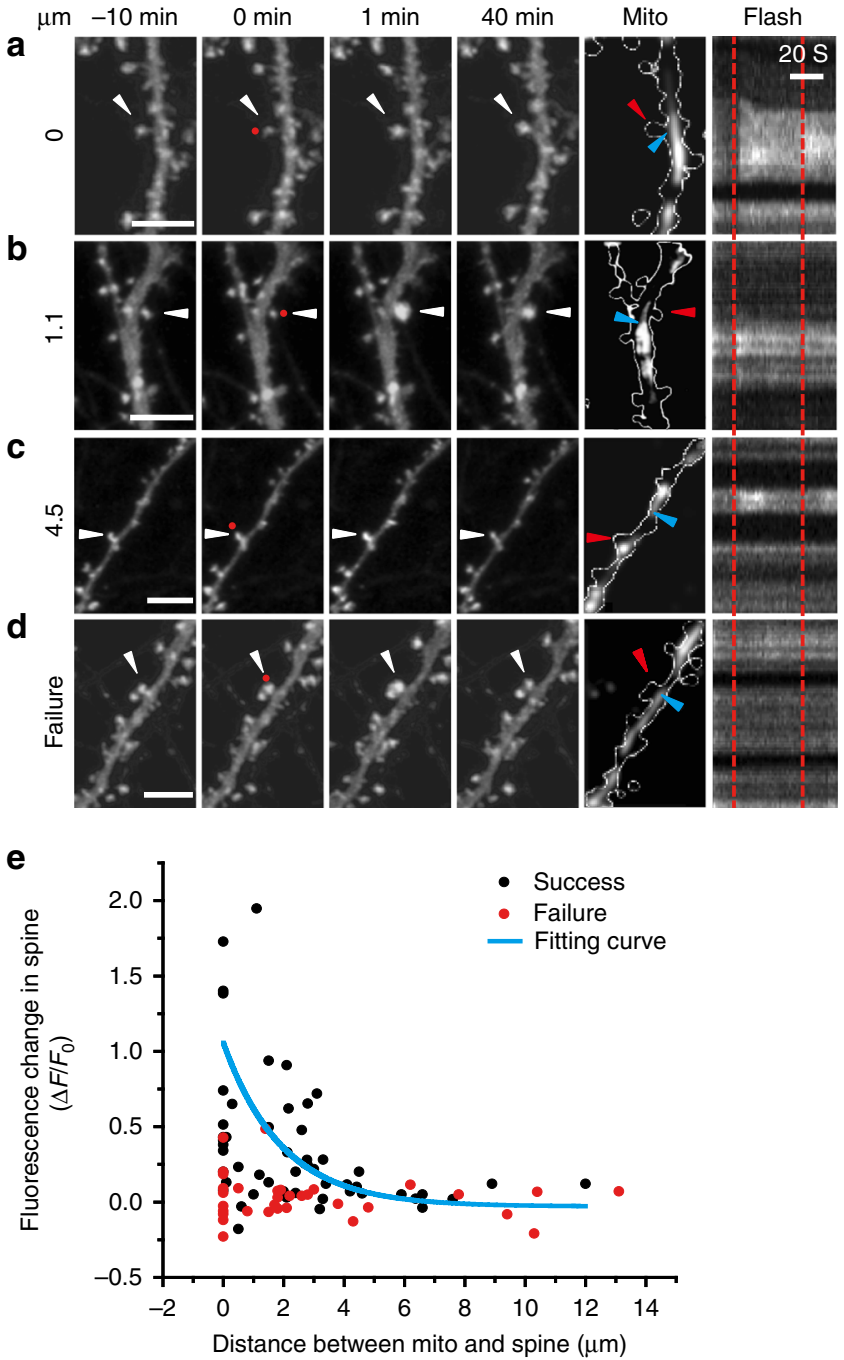

f

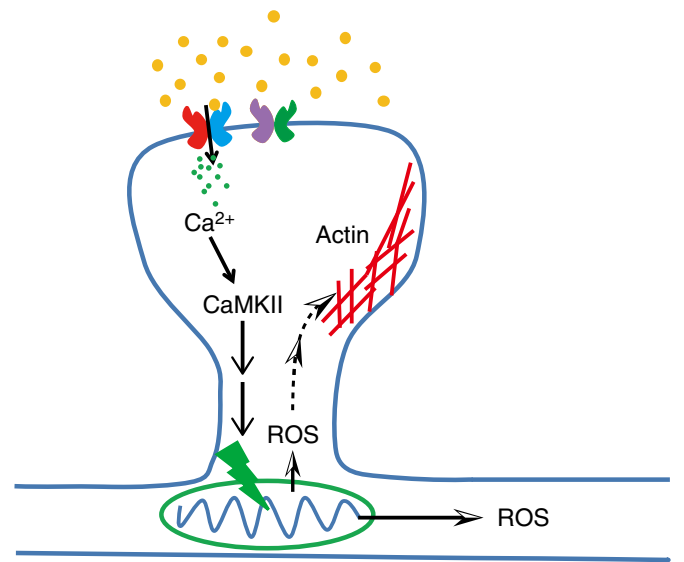

$\Omega$ Spine

NMDA receptor

AMPA receptor

Auv. Mitochondrion

- Glutamate $\quad \mathrm{Ca}^{2+}$

Mitoflash accompanied by transient increases in local ROS, but not calcium, that spread over a few micrometres, in a spatial pattern closely resembling that of mitoflash regulation of sLTP. Therefore, while energy provision by the mitochondria is undoubtedly crucial for long-term synaptic modification, and we cannot fully exclude the possibility that mitoflash is an epiphenomenon during sLTP, it is possible that the role of mitoflashes go beyond bioenergetics. Specifically, we hypothesize that mitoflashes generate local ROS transients to signal for the stabilization of spine sLTP. Indeed, emerging evidence indicates that ROS can play physiological roles in various cellular processes such as regulating synaptic transmission $^{48}$ and modification of the actin cytoskeleton via its interaction with small GTPases ${ }^{34,49}$, and ROS may be directly involved in signaling synaptic plasticity ${ }^{12}, 42,45$. While previous studies have focused on roles of extracellularly and cytosolically generated sustained ROS in LTP induction ${ }^{45}$, our work points to a new scenario in which bursts of mitochondria-generated ROS occur locally and timely to support the expression of sLTP in adjacent spines. Intriguingly, from the LTD-like size reduction and increase in mitoflash occurrence observed in a small portion of spines following glycine stimulation (Fig. 1g), it appears that the mitoflash and perhaps the resultant ROS transient also stabilizes structural changes in the negative direction. More generally, mitoflashes produce packets of ROS signal that are qualitatively distinguishable from basal mitochondrial ROS produced by constitutive electron leakage of the electron transfer chain. Whereas sustained elevation of global ROS is often detrimental, mitoflash-generated ROS can be event-driven, spatially confined, and promptly extinguished. All these salient features point to an appealing cellular mechanism for generating brief and intense ROS pulses, which could in turn activate high-threshold ROS effectors and pathways locally. Such a mechanism that exploits the handy mitochondrial ROS machinery and the ROS signaling toolkits could in principle exist in various cellular contexts and should be evaluated in future studies.

The induction and action of mitoflashes thus may add a novel, spatiotemporally specific mechanism to the picture of the complex and dynamic cellular signaling network underlying synaptic plasticity ${ }^{6,28}$. As such, tuning mitoflash activity might afford an effective means to regulate structural plasticity at synapses. Furthermore, given that more than one spine can share an extended mitochondrion, this spine-mitochondrion communication might provide a basis for a subtle form of "inter-spine association" whereby one spine can facilitate sLTP in another. Such a process could contribute, together with other diffusible signaling mechanisms (e.g., $\mathrm{Ca}^{2+}$ and ATP $)^{8}$, to the clustering of

Fig. 6 Spatial specificity of putative mitoflash signaling in SLTP at dendritic spines. a-d Representative examples of time-dependent spine enlargement with PA-mitoflashes elicited (or attempted) at various distances from the target spine $10 \mathrm{~min}$ after glutamate uncaging in the HW paradigm (See also Supplementary Fig. 6a). Red dots (at 0 min) denote the uncaging spots placed close to spines. White arrowheads denote uncaging target spines. Red and blue arrowheads mark target spines and target mitochondria, respectively. Vertical dashed lines mark the timing of laser pulses. Scale bars, $5 \mu \mathrm{m}$. e Degree of long-term change in spine size as a function of the spinemitochondrion distance for successful PA-mitoflashes (black dots). A single-exponential curve fitting yielded a spatial decay constant of $1.94 \mu \mathrm{m}$. Cases with failed attempts are shown as controls (red dots). $n=12$ neurons and ten batches for both the success (53 spines) and failure groups (34 spines). $\mathbf{f}$ Schematic summary of possible role of mitoflash signaling in the structural plasticity of dendritic spines. Activation of postsynaptic NMDARs causes $\mathrm{Ca}^{2+}$ influx and subsequent CaMKII activation in the spine, which leads to the generation of a local dendritic mitoflash via yet-tobe-delineated processes. Dendritic mitoflashes so activated stabilize spine enlargement likely via a diffusive ROS signal that targets the cytoskeleton 
dendritic spines during learning ${ }^{40,50-52}$. Further studies of the two-way signaling between synapses and mitochondria are likely to reveal new mechanistic insights into synaptic plasticity and mitochondrion-related brain disorders.

\section{Methods}

Isolation and culture of primary hippocampal neurons. All procedures were performed following the guidelines of the Animal Experiments Committees at the University of Science and Technology of China and Peking University.

Primary cultures were prepared using neurons from embryonic rat hippocampus ${ }^{33}$. Briefly, both hippocampi of randomly selected E18 embryos (without distinguishing sex difference) from timed-pregnant rats (Beijing Vital River Laboratory Animal Technology

Co. Ltd., Beijing, China) were removed and digested with $0.25 \%$ trypsin (Sigma-Aldrich, St. Louis, MO) for $15 \mathrm{~min}$ at $37^{\circ} \mathrm{C}$, followed by washing in Hanks' balanced salt solution (HBSS) without $\mathrm{Ca}^{2+}$ and $\mathrm{Mg}^{2+}$ (Thermo Fisher, Waltham, MA) and gentle pipetting. The dissociated neurons were then plated on poly-Llysine (Sigma-Aldrich)-coated glass coverslips in $35-\mathrm{mm}$ petri dishes at $1 \times 10^{5}$ cells $\mathrm{ml}^{-1}$ and kept in serum-free medium (plating medium: Neurobasal medium (Thermo Fisher) supplemented with $2 \%$ B27 (Thermo Fisher), $0.5 \mathrm{mM}$ L-glutaMAX (Thermo Fisher), $37.5 \mathrm{mM} \mathrm{NaCl}$ (Sigma-Aldrich), and $25 \mu \mathrm{M}$ L-glutamic acid (Sigma-Aldrich)) in $5 \% \mathrm{CO}_{2}$ at $37^{\circ} \mathrm{C}$. 4 days after plating, half of the plating medium was exchanged with new medium (Neurobasal medium supplemented with $2 \%$ B27, $0.5 \mathrm{mM}$ L-glutaMAX, $37.5 \mathrm{mM} \mathrm{NaCl}$ ). The medium was then replaced every 4 days.

Plasmid transfection. Cultured neurons were transfected with an mt-cpYFPcontaining plasmid (CaMKII-mt-cpYFP) and a pactin-mCherry-containing plasmid (CaMKII-Bactin-mCherry) at 8-9 DIV (days in vitro) using a calcium phosphate transfection kit (Thermo Fisher). Briefly, neurons cultured on coverslips were transferred to a new petri dish with pre-warmed transfection medium (Neurobasal medium supplemented with 2\% B27, $0.5 \mathrm{mM}$ L-glutaMAX, $37.5 \mathrm{mM}$ $\mathrm{NaCl}, \mathrm{pH}$ 7.4), while the culture medium was preserved in the original dish. CaMKII-mt-cpYFP $(7.5 \mu \mathrm{g})$ and CaMKII- $\beta$ actin-mCherry DNA $(2.0 \mu \mathrm{g})$ along with $15.5 \mu \mathrm{l}$ of $2 \mathrm{moll}^{-1} \mathrm{CaCl}_{2}$ were diluted in $\mathrm{ddH}_{2} \mathrm{O}(125 \mu \mathrm{l}$ in total). The DNA solution was added into $125 \mu \mathrm{l} 2 \times$ HBSS followed by slow pipetting and gentle vortexing with an eighth volume added each time. The mixture was then incubated for 15-20 $\mathrm{min}$ at room temperature and added dropwise to the culture dish with transfection medium. After incubating the neurons for $80 \mathrm{~min}$ in $5 \% \mathrm{CO}_{2}$ at $37^{\circ} \mathrm{C}$, an evenly distributed layer of precipitate particles was observed across the neurons under a $\times 4$ objective. The DNA-Ca ${ }^{2+}$-phosphate precipitates were dissolved with freshly-made dissolution medium (feeding medium with extra $20 \mathrm{mM}$ HEPES, $\mathrm{pH}$ 6.8) and incubated for $4-8 \mathrm{~min}$ at room temperature. Typically, no precipitate particles were then observed. The transfected neurons were then transferred back to their original dishes containing culture medium. The same transfection protocol is used for all the other plasmids transfection. All Imaging experiments were done with 17-20 DIV neurons, except for Fig. 2g-i, which were done with 13-15 DIV neurons.

Electrophysiology and cLTP induction. Whole-cell perforated patch recordings were performed on 13-15 DIV neurons, using amphotericin B for perforation. The glass micropipettes were made on a PC-10 puller (Narishige, Tokyo, Japan), and had resistances of 2-4M 2 . The pipettes were tip-filled with internal solution [containing (in $\mathrm{mM}$ ): potassium gluconate $136.5, \mathrm{KCl} 17.5, \mathrm{NaCl} 9, \mathrm{MgCl}_{2} 1$, HEPES 10, EGTA 0.2, pH 7.20] and then back-filled with internal solution containing $150 \mathrm{ng} \mathrm{ml}^{-1}$ amphotericin B. Neurons were perfused with fresh extracellular bath solution (ECS) [containing (in $\mathrm{mM}$ ): $\mathrm{NaCl} 150, \mathrm{KCl} 3, \mathrm{CaCl}_{2} 3$, HEPES 10, glucose 8 , tetrodotoxin 0.0005 , strychnine 0.001 , and bicuculline methiodide $0.02, \mathrm{pH} 7.30$ ] at a slow rate $\left(0.7 \mathrm{ml} \mathrm{min}^{-1}\right)$ throughout recordings. Miniature synaptic currents were recorded with a patch-clamp amplifier (MultiClamp 700B; Molecular Devices, Sunnyvale, CA) on an inverted microscope (Carl Zeiss Axio Observer, Oberkochen, Germany) with phase optics. For cLTP induction, cultured hippocampal neurons were treated with glycine $(100 \mu \mathrm{M})$ in ECS for $5 \mathrm{~min}$ and then transferred to ECS without glycine. Signals were filtered at $5 \mathrm{kHz}$ and sampled at $10 \mathrm{kHz}$. Data were analyzed with Igor Pro (WaveMetrics, Portland, OR). All experiments were performed at room temperature.

Field electrical stimulation for LTP induction. Primary hippocampal neurons were perfused in extracellular solution containing (in $\mathrm{mM}$ ): $\mathrm{NaCl} 150, \mathrm{KCl} 3, \mathrm{CaCl}_{2} 3$, HEPES $10, \mathrm{MgCl}_{2} 2$, glucose $8, \mathrm{pH} 7.30$, at the speed of $0.7 \mathrm{ml} \mathrm{min}^{-1}$. Electrical field stimulation of about $15 \mathrm{~V} \mathrm{~cm}^{-1}$ was applied through a pair of platinum wires, using $5 \mathrm{~ms}$ voltage pulses delivered from high current capacity stimulators.

Neurons were stimulated with high frequency stimulation (HFS, 3 X 100 pulses at $100 \mathrm{~Hz}, 20 \mathrm{~s}$ apart) to induce LTP.

Imaging mitoflashes and spine morphology. An upright laser-scanning confocal microscope (Zeiss LSM NLO 710, Oberkochen, Germany) equipped with a waterimmersion objective (WPlan-Apochromat $\times 20$, numerical aperture 1.0 , Zeiss) or an inverted confocal microscope (Zeiss LSM NLO 710) equipped with an oil-immersion objective (Plan-Apochromat $\times 40$, numerical aperture 1.3, Zeiss) was used for imaging. Neurons cultured on a coverslip were perfused with extracellular solution containing (in $\mathrm{mM}$ ): $\mathrm{NaCl} \mathrm{150,} \mathrm{KCl} 3, \mathrm{CaCl}_{2} 3$, HEPES 10, D-glucose 8, tetrodotoxin $0.0005, \mathrm{pH} \mathrm{7.4)}$ at rate of $1 \mathrm{ml} \mathrm{min}^{-1}$ in a perfusion chamber. For cLTP induction, $1 \mu \mathrm{M}$ strychnine and $20 \mu \mathrm{M}$ bicuculline methiodide (Sigma Aldrich) were included in the extracellular solution.

Time-lapse images of mt-cpYFP were captured by excitation at $488 \mathrm{~nm}$ (laser power, $2-3 \%$ ) and collecting the emission at $490-550 \mathrm{~nm}$. In typical timeseries recordings of mitoflashes, 350 frames of $512 \times 512$ pixels were collected every $10 \mathrm{~min}$ at $0.10-0.15 \mu \mathrm{m}$ per pixel in bidirectional scanning mode, and the axial resolution was set to $0.8-1.0 \mu \mathrm{m}$. In glutamate-uncaging experiments, 270 frames were collected at 38 frames min $^{-1}$ from 3 to 10 min after uncaging. For mitoSOX (Invitrogen) fluorescence measurement, the indicator $(3 \mu \mathrm{M})$ was loaded at $37^{\circ} \mathrm{C}$ for $20 \mathrm{~min}$ followed by three washes, and cells were imaged by excitation at $543 \mathrm{~nm}$ and collecting the emission at $>585 \mathrm{~nm}$.

We used Image ${ }^{53}$, ZEN (Carl Zeiss), and MATLAB (MathWorks, Inc.) software for image analysis. For mitoflash detection, we analyzed the changes of mt-cpYFP fluorescence intensity in target dendritic mitochondria with Zeiss ZEN software. The standard deviation (SD) of $F / F_{0}$ for the entire trace was calculated, where $F_{0}$ is the average baseline fluorescence intensity. The criteria for a mitoflash event were: (1) peak $\mathrm{F} / \mathrm{F}_{0}>3 \mathrm{SD}$; and (2) full duration at half-maximum $>3 \mathrm{~s}$.

To analyze their structural plasticity, we measured changes in the intensity of actin-mCherry fluorescence in spines. Briefly, 3-D stacks of mCherry images were acquired by excitation at $543 \mathrm{~nm}$ and collecting the emission at $>590 \mathrm{~nm}$ at a series of time points. Typically, 9-10 frames were collected at $0.10-0.12 \mu \mathrm{m}$ per pixel in unidirectional scanning mode at axial intervals of $0.5-0.65 \mu \mathrm{m}$. One 3-D stack of mCherry images was acquired every $10 \mathrm{~min}$, alternating with the mitoflash image acquisition described above. After X-Y drift correction with Image Stabilizer ${ }^{54}$, a time series of the maximal intensity projection was generated, and photobleaching correction was carried out using Bleach Correction ${ }^{55}$. Then, the fluorescence intensity of target spines was analyzed using ImageJ. The fluorescence intensity at different time points was normalized to the basal value (average fluorescence intensity before glycine or uncaging stimulation). The analysis of spine size was blinded to the results of nearby mitoflash events with one author analyzing spine size, and another analyzing mitoflash events separately. The dendrites under severe $Z$-axis drifts were excluded from analysis.

ATP detection during mitoflashes. For simultaneous imaging of local ATP detection and mitoflashes, primary hippocampal neurons were either co-transfected with CaMKII-mt-pHTomato and CaMKII-PercevalHR or, in cells transfected with CaMKII-mt-pHTomato only, loaded with Magnesium Green, $\mathrm{AM}$ (ThemoFisher) prior to imaging, at $5 \mu \mathrm{M}$ for $30 \mathrm{~min}$ and then washed for $3 \times 5$ min. Image acquisition was performed 7 days after transfection, by excitation at 488 and $543 \mathrm{~nm}$ and collecting the emission at 500-530 and $>600 \mathrm{~nm}$, respectively, for CaMKII-PercevalHR and CaMKII-mt-pHTomato co-imaging; by excitation at 488 and $543 \mathrm{~nm}$ and collecting the emission at $500-530 \mathrm{~nm}$ and $>600 \mathrm{~nm}$, respectively, for Magnesium Green and CaMKII-mt-pHTomato co-imaging.

Dendritic ROS detection. The indicator DCF (Invitrogen, Carlsbad, CA) $(5 \mu \mathrm{M})$ was loaded at $37^{\circ} \mathrm{C}$ for $10 \mathrm{~min}$ followed by $3 \times 5 \mathrm{~min}$ washes, and image acquisition was performed by excitation at $488 \mathrm{~nm}$ and collecting the emission at $>500 \mathrm{~nm}$ when imaged alone or $500-530 \mathrm{~nm}$ when co-imaged with mt-pHTomato. To avoid mitochondrial enrichment of DCF, image acquisition was performed only within $30 \mathrm{~min}$ after washes.

Calcium imaging. Neurons were transfected with CaMKII-GCaMP6f on 8-9DIV and used for imaging 1 week later. Image acquisition was performed by excitation at $488 \mathrm{~nm}$ and collecting the emission at $>500 \mathrm{~nm}$ when imaging alone, or $500-530 \mathrm{~nm}$ when co-imaged with mt-pHTomato.

Photo-activation of mitoflashes. Zeiss Multispot Macro was used to photoactivate mitoflashes. The protocol was modified from a previous method ${ }^{44}$ after optimizing the laser wavelength, power, train duration, and number of trains. Briefly, photo-illumination with a 720-nm laser (Ti: sapphire laser, $690-1080 \mathrm{~nm}$, $80 \mathrm{MHz}, 10 \mathrm{~mW}$, Coherent, Santa Clara, CA) was positioned at a diffractionlimited spot on a mitochondrion, with train duration of $1 \mathrm{~ms}$, train number of two, and train interval of $30 \mathrm{~s}$. The probability at which single laser stimulation successfully induced a mitoflash was $\sim 50 \%$. In contrast to the previous work, our photo-activation protocol did not induce mitochondrial swelling and fragmentation (Supplementary Fig. 7), or local ROS or calcium change (Supplementary Figs. 7, 9).

MNI-glutamate uncaging with the two-photon method. MNI-glutamate (Tocris, Bristol, UK) was photolyzed by a $720-\mathrm{nm}$ beam at $8 \mathrm{~mW}$ from a Ti: sapphire laser (Coherent, $690-1080 \mathrm{~nm}$ ). The stimulus duration was $2.5 \mathrm{~ms}$ and the photolysis procedure was controlled using a Zeiss Multispot Macro. We used three different photolysis paradigms. First, for strong uncaging with a high concentration of MNI-glutamate (HS-uncaging), $6 \mathrm{mM}$ MNI-glutamate was used, and 120 
episodes of laser illumination at $2 \mathrm{~Hz}$ were applied to a target spine. Second, for weak uncaging with a high concentration of MNI-glutamate (HW-uncaging), $6 \mathrm{mM}$ MNI-glutamate was used, and 40 episodes at $2 \mathrm{~Hz}$ were applied. Third, for strong uncaging with a low concentration of MNI-glutamate (LS-uncaging), $2 \mathrm{mM}$ MNI-glutamate was used, and 120 episodes at $2 \mathrm{~Hz}$ were applied.

Statistics. Required sample sizes were estimated based on experience of similar experiments. The experiments were not randomized. Data are expressed as mean \pm s.e.m. The paired $t$-test was used to determine the statistical differences between samples at different time points. The Mann-Whitney test was used to determine statistical differences between two groups. For paired $t$-tests, all data were drawn from normally distributed populations. For all statistical tests, tests were two-sided and a $P$ value $<0.05$ was considered statistically significant.

Data availability. All supporting data are available from the authors upon request.

Received: 24 August 2016 Accepted: 28 April 2017

Published online: 26 June 2017

\section{References}

1 Bi, G.-Q. \& Poo, M.-M. Synaptic modification by correlated activity: Hebb's postulate revisited. Annu. Rev. Neurosci. 24, 139-166 (2001).

2 Bliss, T. V. \& Collingridge, G. L. A synaptic model of memory: long-term potentiation in the hippocampus. Nature 361, 31-39 (1993).

3 Hebb, D. O. The Organization of Behavior (Wiley, 1949).

4 Malenka, R. C. \& Nicoll, R. A. Long-term potentiation - a decade of progress. Science 285, 1870-1874 (1999).

5 Derkach, V. A., Oh, M. C., Guire, E. S. \& Soderling, T. R. Regulatory mechanisms of AMPA receptors in synaptic plasticity. Nat. Rev. Neurosci. 8, 101-113 (2007).

6 Nishiyama, J. \& Yasuda, R. Biochemical computation for spine structural plasticity. Neuron 87, 63-75 (2015).

7 Dillon, C. \& Goda, Y. The actin cytoskeleton: integrating form and function at the synapse. Annu. Rev. Neurosci. 28, 25-55 (2005).

8 Harvey, C. D., Yasuda, R., Zhong, H. \& Svoboda, K. The spread of ras activity triggered by activation of a single dendritic spine. Science 321, 136-140 (2008).

9 Zhai, S., Ark, E. D., Parra-Bueno, P. \& Yasuda, R. Long-distance integration of nuclear ERK signaling triggered by activation of a few dendritic spines. Science 342, 1107-1111 (2013).

10 Colgan, L. A. \& Yasuda, R. Plasticity of dendritic spines: subcompartmentalization of signaling. Annu. Rev. Physiol. 76, 365 (2014).

11 Harris, J. J., Jolivet, R. \& Attwell, D. Synaptic energy use and supply. Neuron 75, 762-777 (2012).

12 Cheng, A., Hou, Y. \& Mattson, M. P. Mitochondria and neuroplasticity. ASN Neuro 2, AN20100019 (2010).

13 Kang, J.-S. et al. Docking of axonal mitochondria by syntaphilin controls their mobility and affects short-term facilitation. Cell 132, 137-148 (2008).

$14 \mathrm{Li}$, Z. et al. Caspase-3 activation via mitochondria is required for long-term depression and AMPA receptor internalization. Cell 141, 859-871 (2010).

$15 \mathrm{Li}, \mathrm{Z}$., Okamoto, K.-I., Hayashi, Y. \& Sheng, M. The importance of dendritic mitochondria in the morphogenesis and plasticity of spines and synapses. Cell 119, 873-887 (2004).

16 Wang, W. et al. Superoxide flashes in single mitochondria. Cell 134, 279-290 (2008).

17 Santo-Domingo, J., Giacomello, M., Poburko, D., Scorrano, L. \& Demaurex, N. OPAl promotes $\mathrm{pH}$ flashes that spread between contiguous mitochondria without matrix protein exchange. EMBO J. 32, 1927-1940 (2013).

18 Wei-LaPierre, L. et al. Respective contribution of mitochondrial superoxide and $\mathrm{pH}$ to mitochondria-targeted circularly permuted yellow fluorescent protein (mt-cpYFP) flash activity. J. Biol. Chem. 288, 10567-10577 (2013).

19 Zhang, X. et al. Superoxide constitutes a major signal of mitochondrial superoxide flash. Life Sci. 93, 178-186 (2013).

20 Pouvreau, S. Superoxide flashes in mouse skeletal muscle are produced by discrete arrays of active mitochondria operating coherently. PLoS One 5, e13035 (2010)

21 Shen, E.-Z. et al. Mitoflash frequency in early adulthood predicts lifespan in Caenorhabditis elegans. Nature 508, 128-132 (2014).

22 Fang, H. et al. Imaging superoxide flash and metabolism-coupled mitochondrial permeability transition in living animals. Cell Res. 21, 1295-1304 (2011).

$23 \mathrm{Hou}$, Y. et al. Mitochondrial superoxide production negatively regulates neural progenitor proliferation and cerebral cortical development. Stem Cells $\mathbf{3 0}$, 2535-2547 (2012).

24 Ying, Z. et al. Transient activation of mitoflashes modulates nanog at the early phase of somatic cell reprogramming. Cell Metab. 23, 220-226 (2016).
$25 \mathrm{Lu}$, W. et al. Activation of synaptic NMDA receptors induces membrane insertion of new AMPA receptors and LTP in cultured hippocampal neurons. Neuron 29, 243-254 (2001).

26 Colicos, M. A., Collins, B. E., Sailor, M. J. \& Goda, Y. Remodeling of synaptic actin induced by photoconductive stimulation. Cell 107, 605-616 (2001).

27 Bi, G.-Q. \& Poo, M.-M. Synaptic modifications in cultured hippocampal neurons: Dependence on spike timing, synaptic strength, and postsynaptic cell type. J. Neurosci. 18, 10464-10472 (1998).

28 Wang, H.-X., Gerkin, R. C., Nauen, D. W. \& Bi, G.-Q. Coactivation and timingdependent integration of synaptic potentiation and depression. Nat. Neurosci. 8 , 187-193 (2005).

29 Bosch, M. et al. Structural and molecular remodeling of dendritic spine substructures during long-term potentiation. Neuron 82, 444-459 (2014).

30 Straub, C. \& Sabatini, B. L. How to grow a synapse. Neuron 82, 256-257 (2014).

31 Harward, S. C. et al. Autocrine BDNF-TrkB signalling within a single dendritic spine. Nature 538, 99-103 (2016).

32 Herring, B. E. \& Nicoll, R. A. Long-term potentiation: from CaMKII to AMPA receptor trafficking. Annu. Rev. Physiol. 78, 351-365 (2016).

33 Zhang, J.-C., Lau, P.-M. \& Bi, G.-Q. Gain in sensitivity and loss in temporal contrast of STDP by dopaminergic modulation at hippocampal synapses. Proc. Natl. Acad. Sci. USA 106, 13028-13033 (2009).

34 Breckwoldt, M. O. et al. Multiparametric optical analysis of mitochondrial redox signals during neuronal physiology and pathology in vivo. Nat. Med. 20, 555-60 (2014).

35 Lisman, J., Yasuda, R. \& Raghavachari, S. Mechanisms of CaMKII action in long-term potentiation. Nat. Rev. Neurosci. 13, 169-182 (2012).

36 Engert, F. \& Bonhoeffer, T. Dendritic spine changes associated with hippocampal long-term synaptic plasticity. Nature 399, 66-70 (1999).

37 Oh, W. C., Parajuli, L. K. \& Zito, K. Heterosynaptic structural plasticity on local dendritic segments of hippocampal CA1 neurons. Cell Rep. 10, 162-169 (2015).

38 Matsuzaki, M., Honkura, N., Ellis-Davies, G. C. R. \& Kasai, H. Structural basis of long-term potentiation in single dendritic spines. Nature 429, 761-766 (2004).

39 Zito, K., Knott, G., Shepherd, G. M., Shenolikar, S. \& Svoboda, K. Induction of spine growth and synapse formation by regulation of the spine actin cytoskeleton. Neuron 44, 321-334 (2004).

40 Govindarajan, A., Israely, I., Huang, S. Y. \& Tonegawa, S. The dendritic branch is the preferred integrative unit for protein synthesis-dependent LTP. Neuron 69, 132-146 (2011).

41 Lee, S.-J. R., Escobedo-Lozoya, Y., Szatmari, E. M. \& Yasuda, R. Activation of CaMKII in single dendritic spines during long-term potentiation. Nature 458, 299-304 (2009).

42 Murakoshi, H., Wang, H. \& Yasuda, R. Local, persistent activation of Rho GTPases during plasticity of single dendritic spines. Nature 472, 100-104 (2011).

43 Wang, X. et al. Protons trigger mitochondrial flashes. Biophys. J. 111, 386-394 (2016).

44 Shi, F. et al. Mitochondrial swelling and restorable fragmentation stimulated by femtosecond laser. Biomed. Opt. Express 6, 4539-4545 (2015).

45 Massaad, C. A. \& Klann, E. Reactive oxygen species in the regulation of synaptic plasticity and memory. Antioxid. Redox Signal. 14, 2013-2054 (2011).

46 Kamsler, A. \& Segal, M. Hydrogen peroxide modulation of synaptic plasticity. J. Neurosci. 23, 269-276 (2003).

47 Wei, L. et al. Mitochondrial superoxide flashes: metabolic biomarkers of skeletal muscle activity and disease. FASEB J. 25, 3068-3078 (2011).

48 Accardi, M. V. et al. Mitochondrial reactive oxygen species regulate the strength of inhibitory GABA-mediated synaptic transmission. Nat. Commun. 5, 3168 (2014).

49 Muliyil, S. \& Narasimha, M. Mitochondrial ROS regulates cytoskeletal and mitochondrial remodeling to tune cell and tissue dynamics in a model for wound healing. Dev. Cell. 28, 239-252 (2014).

$50 \mathrm{Fu}, \mathrm{M} ., \mathrm{Yu}, \mathrm{X}$., Lu, J. \& Zuo, Y. Repetitive motor learning induces coordinated formation of clustered dendritic spines in vivo. Nature 483, 92-95 (2012).

51 Govindarajan, A., Kelleher, R. J. \& Tonegawa, S. A clustered plasticity model of long-term memory engrams. Nat. Rev. Neurosci. 7, 575-583 (2006).

52 Rogerson, T. et al. Synaptic tagging during memory allocation. Nat. Rev. Neurosci. 15, 157-169 (2014).

53 Schneider, C. A., Rasband, W. S. \& Eliceiri, K. W. NIH Image to ImageJ: 25 years of image analysis. Nat. Methods 9, 671-675 (2012).

$54 \mathrm{Li}, \mathrm{K}$. The image stabilizer plugin for ImageJ. http://www.cs.cmu.edu/ kangli/ code/Image_Stabilizer.html (2008).

55 Rietdorf, J. The Bleach Correction macro for ImageJ. http://www.embl.de/ eamnet/html/bleach_correction.html (2005).

\section{Acknowledgements}

We thank Bin Zhang for help with culture preparation, Lei Qi and Jigui Zhang for the actin-mCherry plasmid construction, Xin Gong for the mt-pHTomato plasmid construction, and Drs Mu-ming Poo and IC Bruce for helpful comments on the manuscript. This work was supported in part by the Strategic Priority Research Program of the Chinese Academy of Sciences (XDB02050000), the National Basic Research 
Program of China (2013CB835100, 2013CB531200 and 2016YFA0500403), and the National Science Foundation of China $(31130067,31521062)$.

\section{Author contributions}

H.C. and G.-Q.B. conceived the project, and G.-Q.B., H.C., X.W. and P.-M.L. supervised the project. Z.-X.F. and X.T. designed and implemented the experiments, collected, processed and analyzed data, and drafted the manuscript. H.F. contributed to early experiments. G.-Q.B., H.C., X.W., P.-M.L., Z.-X.F. and X.T. wrote the paper. All authors participated in discussion and critically reviewed the manuscript.

\section{Additional information}

Supplementary Information accompanies this paper at doi:10.1038/s41467-017-00043-3.

Competing interests: The authors declare no competing financial interests.

Reprints and permission information is available online at http://npg.nature.com/ reprintsandpermissions/
Publisher's note: Springer Nature remains neutral with regard to jurisdictional claims in published maps and institutional affiliations.

(c) (i) Open Access This article is licensed under a Creative Commons Attribution 4.0 International License, which permits use, sharing, adaptation, distribution and reproduction in any medium or format, as long as you give appropriate credit to the original author(s) and the source, provide a link to the Creative Commons license, and indicate if changes were made. The images or other third party material in this article are included in the article's Creative Commons license, unless indicated otherwise in a credit line to the material. If material is not included in the article's Creative Commons license and your intended use is not permitted by statutory regulation or exceeds the permitted use, you will need to obtain permission directly from the copyright holder. To view a copy of this license, visit http://creativecommons.org/ licenses/by/4.0/.

(c) The Author(s) 2017 